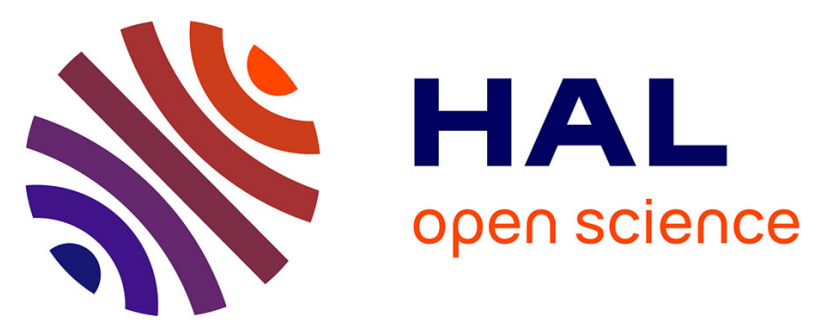

\title{
Value of Relative Myocardial Perfusion at MRI for Fractional Flow Reserve-Defined Ischemia: A Pilot Study
}

Olivier Ghekiere, Jean-Nicolas Dacher, Willem Dewilde, Isabelle Mancini, Wilfried Cools, Piet K Vanhoenacker, Paul Dendale, Patrizio Lancellotti, Albert de Roos, Alain Nchimi

\section{To cite this version:}

Olivier Ghekiere, Jean-Nicolas Dacher, Willem Dewilde, Isabelle Mancini, Wilfried Cools, et al.. Value of Relative Myocardial Perfusion at MRI for Fractional Flow Reserve-Defined Ischemia: A Pilot Study. American Journal of Roentgenology, 2019, 212 (5), pp.1 - 8. 10.2214/AJR.18.20469 . inserm02457256

\section{HAL Id: inserm-02457256 https://www.hal.inserm.fr/inserm-02457256}

Submitted on 19 Feb 2020

HAL is a multi-disciplinary open access archive for the deposit and dissemination of scientific research documents, whether they are published or not. The documents may come from teaching and research institutions in France or abroad, or from public or private research centers.
L'archive ouverte pluridisciplinaire HAL, est destinée au dépôt et à la diffusion de documents scientifiques de niveau recherche, publiés ou non, émanant des établissements d'enseignement et de recherche français ou étrangers, des laboratoires publics ou privés. 


\section{Value of Relative Myocardial Perfusion at MRI for Fractional Flow Reserve-Defined Ischemia: A Pilot Study}

Olivier Ghekiere ${ }^{1,2,3}$

Jean-Nicolas Dacher ${ }^{4}$

Willem Dewilde ${ }^{5}$

Isabelle Mancini ${ }^{1}$

Wilfried Cools ${ }^{6}$

Piet K. Vanhoenacker ${ }^{7}$

Paul Dendale ${ }^{3,8}$

Patrizio Lancellotti 9,10

Albert de Roos ${ }^{11}$

Alain Nchimi ${ }^{9}, 12$
Keywords: fractional flow reserve, ischemia, MRI, myocardial, perfusion

doi.org/10.2214/AJR.18.20469

Received July 30, 2018; accepted after revision November 2, 2018.

\section{AJR 2019; 212:1-8}

0361-803X/19/2125-1

(c) American Roentgen Ray Society
OBJECTIVE. Correcting the perfusion in areas distal to coronary stenosis (risk) according to that of normal (remote) areas defines the relative myocardial perfusion index, which is similar to the fractional flow reserve (FFR) concept. The aim of this study was to assess the value of relative myocardial perfusion by MRI in predicting lesion-specific inducible ischemia as defined by FFR.

MATERIALS AND METHODS. Forty-six patients (33 men and 13 women; mean [ $\pm \mathrm{SD}]$ age, $61 \pm 9$ years) who underwent adenosine perfusion MRI and FFR measurement distal to 49 coronary artery stenoses during coronary angiography were retrospectively evaluated. Subendocardial time-enhancement maximal upslopes, normalized by the respective left ventricle cavity upslopes, were obtained in risk and remote subendocardium during adenosine and rest MRI perfusion and were correlated to the FFR values.

RESULTS. The mean FFR value was $0.84 \pm 0.09$ (range, $0.60-0.98$ ) and was less than or equal to 0.80 in $31 \%$ of stenoses $(n=15)$. The relative subendocardial perfusion index (risk-toremote upslopes) during hyperemia showed better correlations with the FFR value $(r=0.59)$ than the uncorrected risk perfusion parameters (i.e., both the upslope during hyperemia and the perfusion reserve index [stress-to-rest upslopes]; $r=0.27$ and 0.29 , respectively). A cutoff value of 0.84 of the relative subendocardial perfusion index had an ROC AUC of 0.88 to predict stenosis at an FFR of less than or equal to 0.80 .

CONCLUSION. Using adenosine perfusion MRI, the relative myocardial perfusion index enabled the best prediction of FFR-defined lesion-specific myocardial ischemia. This index could be used to noninvasively determine the need for revascularization of known coronary stenoses.

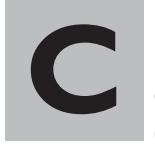
atheter-based fractional flow reserve (FFR) measurement is a reference for the functional significance of lesion-specific coronary artery stenosis, because FFR less than or equal to 0.8 identifies stenosis inducing ischemia and requiring revascularization in clinical practice. The concept of FFR was validated as a relative flow reserve-that is, the ratio of hyperemic flow in a stenotic coronary artery to the hyperemic flow in a normal coronary artery. Therefore, it standardizes inflow conditions, avoiding the confounding effects of microvascular disease and collateral flow [1-4].

Cardiac stress perfusion MRI assesses noninvasively the myocardial vascular sup-
'Department of Radiology, Centre Hospitalier Chrétien, Liège, Belgium.

${ }^{2}$ Department of Radiology, Jessa Ziekenhuis, Hasselt, Belgium.

${ }^{3}$ Faculty of Medicine and Life Sciences, Hasselt University, Hasselt, Belgium.

${ }^{4}$ Department of Radiology, University Hospital, Rouen, France.

${ }^{5}$ Department of Cardiology, Imelda Hospital, Bonheiden, Belgium.

${ }^{6}$ I-BioStat, Centre for Statistics, Hasselt University, Hasselt, Belgium.

${ }^{7}$ Department of Radiology, OLV Ziekenhuis, Aalst, Belgium.

${ }^{8} \mathrm{Heart}$ Center Hasselt, Jessa Ziekenhuis, Hasselt, Belgium.

${ }^{9}$ Department of Cardiology, Heart Valve Clinic, GIGA Cardiovascular Sciences, University of Liège Hospital, CHU Sart Tilman, Liège, Belgium.

${ }^{10} \mathrm{Gruppo}$ Villa Maria Care and Research, Anthea Hospital, Bari, Italy.

${ }^{11}$ Department of Radiology, Leiden University Medical Center, Leiden, The Netherlands.

${ }^{12}$ Department of Cardiovascular Imaging, Centre Hospitalier de Luxembourg, 4 Rue Ernest Barblé, L-1210, Luxembourg. Address correspondence to A. Nchimi (alainnchimi@gmail.com). 
ply by visual, semiquantitative, and quantitative analyses (usually by analyzing the myocardial enhancement curves). Perfusion MRI has been validated on myocardial ischemia models using radiolabeled microspheres. Excellent correlation was shown between the mainstream perfusion defect during maximal hyperemia and microsphere deposition distal to coronary stenoses [5, 6]. In practice, quantitative perfusion MRI considers stressonly or stress-to-rest (myocardial perfusion reserve index) upslopes for the diagnosis of inducible perfusion defects. The correlations of these perfusion parameters and FFR value in the feeding artery, albeit significant, show dispersion [7-11].

The ratio of myocardial enhancement upslopes distal to a coronary stenosis (risk) to that of normally perfused (remote) areas defines the relative perfusion index, which also has shown good correlation with microsphere deposition in a seminal animal study [6]. Although this approach is similar to the FFR principle [2], to our knowledge, it has not been used in humans so far. Therefore, we hypothesized that this index will enhance the correlation with FFR, potentially reducing the need for invasive procedures to determine the suitability of coronary revascularization. The purpose of this study is to assess the value of the relative perfusion index on adenosine MRI in predicting inducible myocardial ischemia, as defined by impaired FFR, on a per-lesion basis.

\section{Materials and Methods \\ Patient Selection and Study Protocol}

This retrospective study protocol was approved by the institutional ethics committee of Centre Hospitalier Chrètien, and patients provided written informed consent. We selected all patients from our hospital database during a 3-year period who were older than 18 years with no history of myocardial infarction and who had undergone coronary angiography and FFR measurement as the final diagnostic tests for stable angina within
4 months of an adenosine perfusion MRI, without any endovascular or surgical intervention in the interim. All the usual contraindications to both examinations were considered. This initial search yielded 57 patients. A first round of review of the imaging data and patient charts was performed by a senior staff radiologist with 10 years of experience in cardiovascular imaging. Patients with transmural myocardial infarct on late gadoliniumenhanced MRI $(n=5)$, those with more than one stenosis with a greater than $70 \%$ diameter reduction in different vascular territories $(n=3)$, and those with more than one stenosis with a greater than $70 \%$ diameter reduction on the same artery $(n=3)$ on quantitative coronary angiography were excluded to avoid microvasculature heterogeneity, possible hemodynamic interactions between coronary territories, and confounding effects of successive stenosis on the flow patterns, respectively. These selection steps allowed the inclusion of 46 patients. Within the study period, three patients had FFR measurement on two stenoses in a different coronary artery. Therefore, a total of 49 stenoses (i.e., lesion-specific risk areas) were evaluated by adenosine perfusion MRI and FFR (Fig. 1).

\section{MRI Protocol}

MRI examinations were performed on a 1.5-T MRI scanner (Avanto, Siemens Healthcare) under continuous heart rate and blood pressure monitoring. All patients were asked to suspend the intake of $\beta$-blockers and competitive antagonists of adenosine (caffeinated beverages) 24 hours before the examination.

Stress perfusion MRI was performed during maximal vasodilatation (i.e., 3 minutes after beginning the injection of $140 \mu \mathrm{mol} / \mathrm{kg} / \mathrm{min}$ of adenosine [Adenocor, Sanofi-Aventis]) using selective saturation-prepared T1-weighted steady-state free precession slices. Image acquisition started simultaneously with the injection of $0.1 \mathrm{mmol} / \mathrm{kg}$ of body weight of a gadolinium chelate (gadodiamide; Omniscan, GE Healthcare) and a 30-mL saline flush; both were given at an injection rate of $4 \mathrm{~mL} / \mathrm{s}$. During a single breath-hold, 50 measurements of the three slices per heartbeat were acquired using a linear sampling of the k-space. The preparation time for each slice, measured to the center of the k-space, was 110 milliseconds. Saturation was obtained with a simple hard pulse with a $90^{\circ}$ nominal flip angle followed by a gradient crusher. Five dummy cycles with a linear flip angle were used to reduce the steady-state free precession signal oscillations. We used symmetric echoes with a TR/TE of 2.2/1.1 and a flip angle of $50^{\circ}$; the resulting acquisition time per image was 178 milliseconds. The bandwidth was $1371 \mathrm{~Hz} /$ pixel, and the matrix size was $192 \times 115$, resulting in an image resolution of $3.5 \times 2.1 \times 8.0 \mathrm{~mm}$. The phase-encoding direction was left-to-right, and the FOV was adjusted to the subjects' size to avoid folding artifacts.

Approximately 10 minutes after the start of the injection of contrast agent, myocardial late gadolinium enhancement imaging was performed using breath-hold phase-sensitive inversion recovery sequences. Then, resting perfusion MR images were acquired under the same technical conditions as the adenosine perfusion MRI.

\section{Semiquantitative Perfusion MRI Analysis}

Semiquantitative analyses were performed using dedicated software (cardiac engine-perfusion module, syngo.via VA30, Siemens Healthcare) after correction of respiratory motion with navigator-guided motion correction (MOCO module, syngo.via VA30, Siemens Healthcare).

First, a visual analysis was performed in consensus by two observers who had full knowledge of the location of the coronary stenosis but were blinded to the FFR data. These observers determined the risk area, taking the coronary dominancy into account. The segment with the greatest transmural extent of the stress-induced myocardial perfusion defect was considered for further measurements when the risk area involved more than one segment of the left ventricle representation [12]. This segment was equally divided into subendocardial and subepicardial regions by outlining the endocardial and epicardial borders. Special emphasis was placed to avoid dark rim artifacts and adjacent tissue and blood from these tracings. Similar steps were performed for a remote myo-

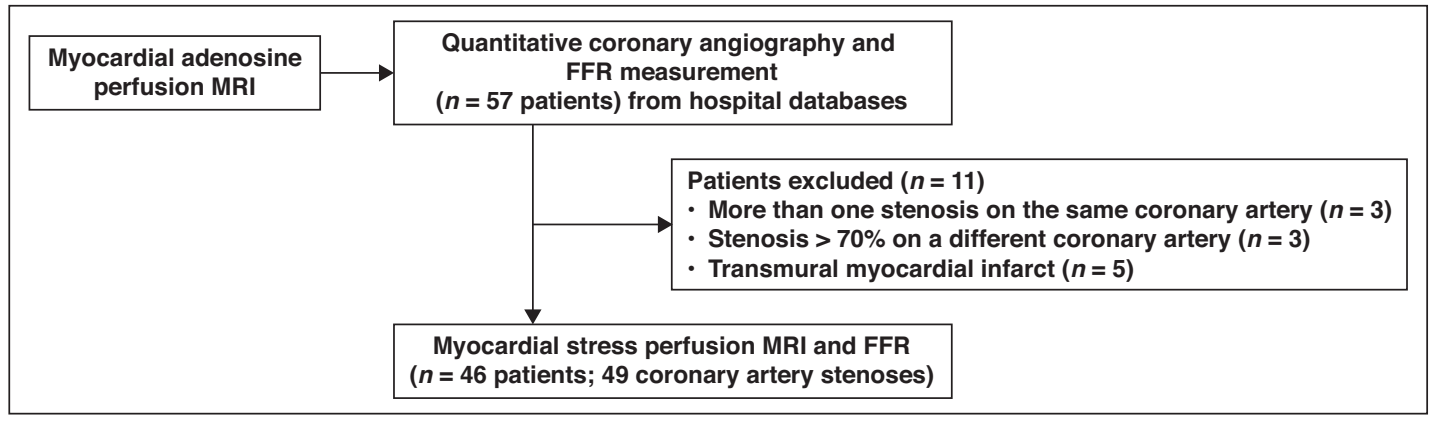

Fig. 1-Patient selection flowchart. FFR = fractional flow reserve. 


\section{Perfusion MRI of FFR-Defined Ischemia}

cardial segment with no greater than $40 \%$ diameter reduction stenosis on the supplying artery.

Then, for each subendocardial risk and remote myocardial segment, the mean maximal initial upslope of the contrast enhancement phase was normalized by its respective left ventricle cavity upslope (measured under a circular ROI of $10 \mathrm{~mm}^{2}$ in the center of the cavity) and recorded in both stress and rest conditions [13] (Fig. 2). If necessary, manual correction was made to adjust the ROI placement.

When no myocardial-inducible defect was visualized, the risk area was defined distal to the anatomic location of the coronary stenosis, and the remaining steps were performed as when a perfusion defect could be visually detected. In patients with more than one coronary stenosis, each perfusion defect was assessed separately.

The following three subendocardial upslopederived perfusion indexes were stored for further analysis: the uncorrected risk upslopes during stress, the myocardial perfusion reserve index (stress-to-rest upslopes), and the relative myocardial perfusion index (risk-to-remote upslopes during stress), as described in Figure 1.

\section{Coronary Angiography and Fractional Flow Reserve Measurements}

Coronary angiography and FFR measurements were performed by an interventional cardiologist, using previously described standard procedures [14]. FFR measurement was performed after placement of a 0.014-inch pressure sensor-tipped coronary angioplasty guidewire across the diseased artery (FloWire Doppler Guide Wire, Volcano). FFR was determined as the ratio of the mean distal coronary to the mean aortic pressure during maximal myocardial hyperemia (i.e., 3060 seconds after the intracoronary injection of 15 $20 \mathrm{mg}$ of papaverine [Sterop], $100 \mathrm{mg} / 3 \mathrm{~mL}$ ).

\section{Statistical Analysis}

Statistical analyses were performed using $\mathrm{R}$ software (version 3.2.3, R Foundation for Statistical Computing). Continuous data with a normal distribution are expressed as mean $\pm \mathrm{SD}$. Where applicable, group comparisons for continuous variables were performed with paired two-tailed $t$ tests and chi-square tests. Pairwise correlations were calculated between all maximal upslopes to investigate the extent to which multicollinearity must be handled. Pairwise correlations were calculated between the FFR value, the upslope in the risk area during stress, the subendocardial perfusion reserve index, and the relative subendocardial perfusion index. Correlation coefficients of $0-0.19,0.20-0.39,0.40-0.59,0.60-0.79$, and $0.80-1$ indicated very weak, weak, moderate, strong and very strong correlations, respective- ly. Diagnostic values for FFR less than or equal to 0.80 of these perfusion MRI indexes are expressed as the sensitivity, specificity, positive predictive value, negative predictive value, likelihood ratios, and accuracy. Diagnostic values are given with their $95 \%$ CIs. A $p<0.05$ was considered to express a statistically significant difference.
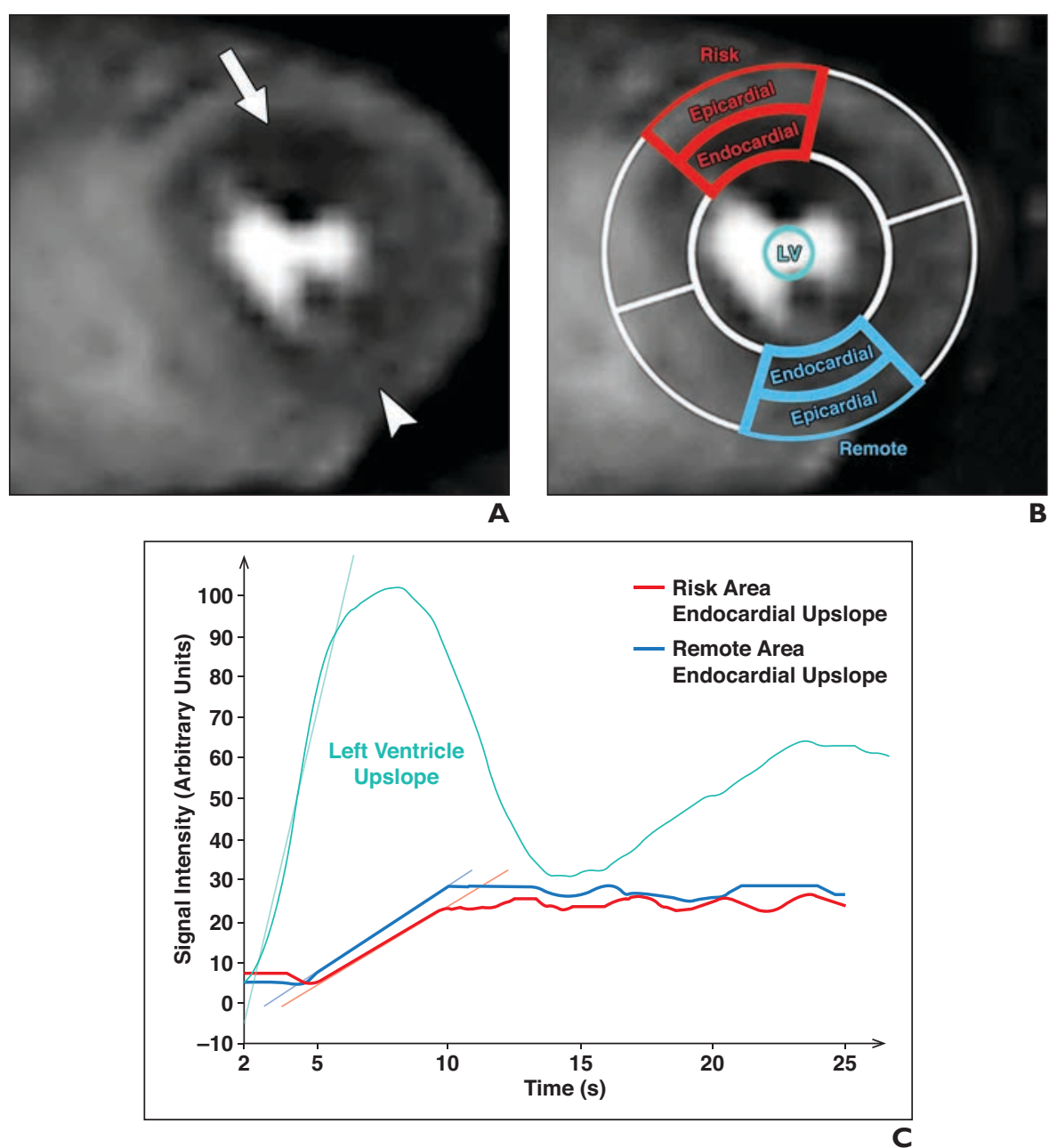

Fig. 2-64-year-old woman with stenosis of midportion of left anterior descending artery.

A-F, Peak myocardial enhancement on stress perfusion MR image shows low signal intensity in segment 7 (arrow, A) of anterior midleft ventricular (risk) myocardium, whereas inferior (remote) myocardium (arrowhead, A) and both areas on rest perfusion MR image (D) enhanced homogeneously. No abnormal enhancement was present on late-enhancement image (not shown). Equally divided subendocardial (bold lines) and subepicardial (thin lines) ROIs are drawn in risk (red) and remote (blue) myocardial segments of left ventricle (LV) after outlining endocardial and epicardial borders of myocardium on peak enhancement during maximal hyperemia (B). Same indicators are present (dashed lines; risk, red; remote, blue) on rest peak myocardial enhancement image (E). After extending these ROIs to whole frames, corresponding subendocardial dynamic contrast enhancement upslope curves (i.e., maximal upslope value of contrast enhancement) were obtained during adenosine (C) and rest (F) perfusion MRI. After normalization by respective left ventricle cavity upslopes, subendocardial upslope in risk myocardium during stress perfusion, perfusion reserve index (i.e., stress-to-rest upslopes), and relative perfusion index (i.e., risk-to-remote upslopes during stress perfusion) were available for further analysis. Lighter diagonal lines denote maximal enhancement upslopes.

(Fig. 2 continues on next page) 

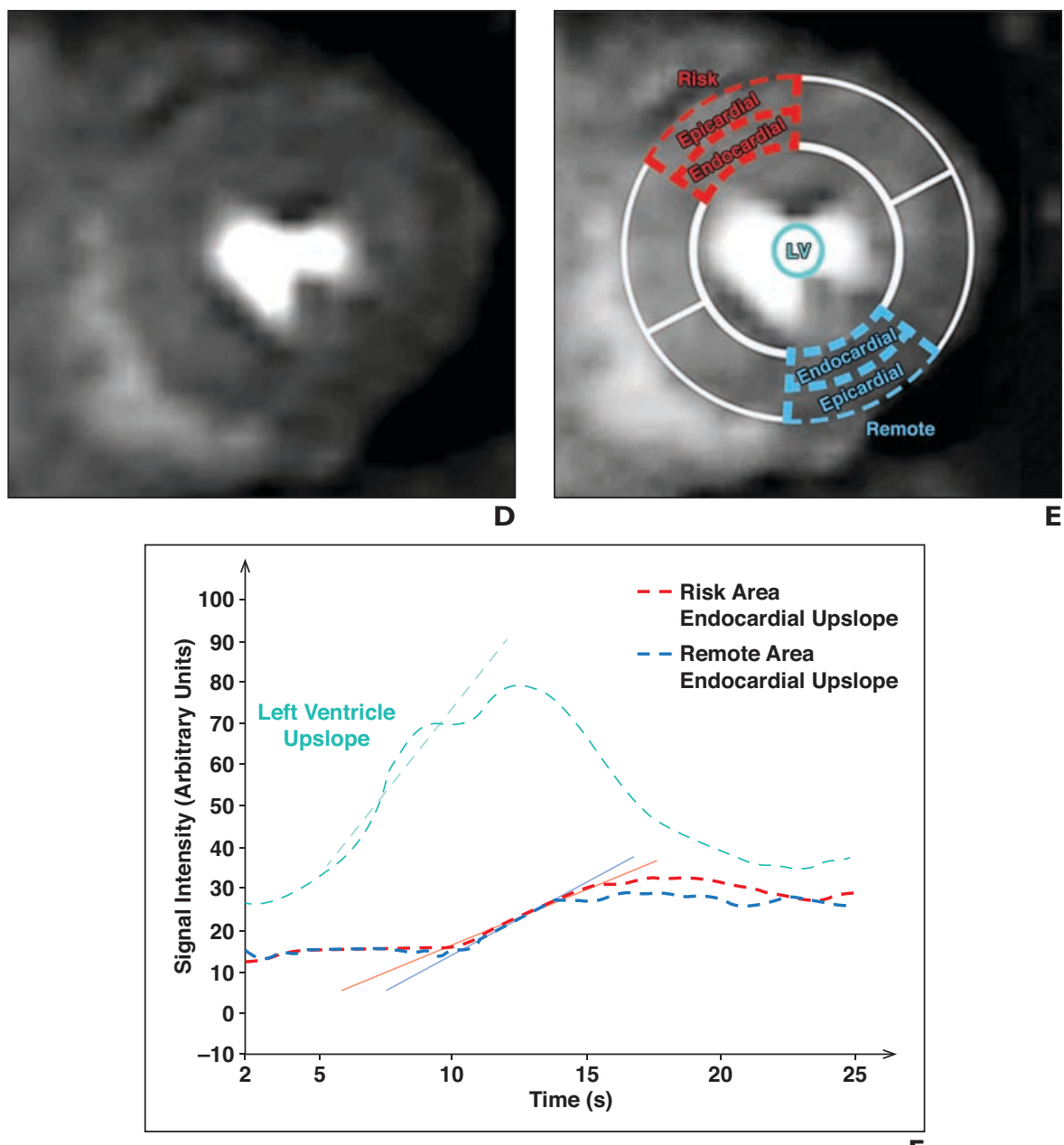

Fig. 2 (continued) -64-year-old woman with stenosis of midportion of left anterior descending artery.

A-F, Peak myocardial enhancement on stress perfusion MR image shows low signal intensity in segment 7 (arrow, A) of anterior midleft ventricular (risk) myocardium, whereas inferior (remote) myocardium (arrowhead, A) and both areas on rest perfusion MR image (D) enhanced homogeneously. No abnormal enhancement was present on late-enhancement image (not shown). Equally divided subendocardial (bold lines) and subepicardial (thin lines) ROIs are drawn in risk (red) and remote (blue) myocardial segments of left ventricle (LV) after outlining endocardial and epicardial borders of myocardium on peak enhancement during maximal hyperemia (B). Same indicators are present (dashed lines; risk, red; remote, blue) on rest peak myocardial enhancement image (E). After extending these ROIs to whole frames, corresponding subendocardial dynamic contrast enhancement upslope curves (i.e., maximal upslope value of contrast enhancement) were obtained during adenosine $(\mathbf{C})$ and rest (F) perfusion MRI. After normalization by respective left ventricle cavity upslopes, subendocardial upslope in risk myocardium during stress perfusion, perfusion reserve index (i.e., stress-to-rest upslopes), and relative perfusion index (i.e., risk-to-remote upslopes during stress perfusion) were available for further analysis. Lighter diagonal lines denote maximal enhancement upslopes.

risk factors are given in Table 1. The mean interval between MRI and FFR measurement was $23.2 \pm 25.9$ days (range, $0-110$ days). The 49 evaluated lesions had a mean percentage of diameter reduction of $56 \% \pm 7 \%$ on quantitative coronary angiography, including 12 (24\%) on the right coronary artery, one (2\%) on the left main coronary artery, 28 (57\%) on the left anterior descending artery, and eight $(16 \%)$ on the left circumflex coronary artery. The mean FFR value in all stenoses was $0.84 \pm 0.09$ (range, $0.60-0.98) ; 31 \%(n=15)$ of the lesions had an FFR less than or equal to 0.80 , with a mean percentage diameter reduc- tion of $59 \% \pm 7.6 \%$ (range, $42-70 \%$ ). No adverse events were observed during MRI, coronary angiography, and FFR measurements.

\section{Correlations Between Fractional Flow Reserve and Adenosine Perfusion MRI}

Table 2 and Figure 3 show the relationship between the FFR values and the three evaluated perfusion MRI indexes. Both the uncorrected risk subendocardial upslope $(r=$ $0.27 ; p=0.06)$ and the perfusion reserve $(r=$ $0.29 ; p=0.04)$ correlated very weakly with the FFR values. The relative perfusion index revealed a significantly improved correlation with the FFR values $(r=0.59 ; p<0.001)$

The AUC values were $0.69,0.67$, and 0.88 for the maximal uncorrected upslope, the subendocardial perfusion reserve index, and the relative subendocardial perfusion index, respectively (Fig. 4). The relative subendocardial perfusion index yielded a significantly higher diagnostic accuracy to predict FFR less than or equal to 0.80 than the maximal uncorrected upslope ( $88 \%$ vs $67 \% ; p<0.001)$ and the subendocardial perfusion reserve index ( $88 \%$ vs $59 \% ; p<0.001)$ (Table 3). Using the cutoff value of 0.84 , the relative subendocardial perfusion index had three false-positives and three false-negatives in predicting FFR less than or equal to 0.80 . Interestingly, all false-negative cases exhibited the socalled splenic switch-off, which occurs on stress perfusion MRI when the stressor fails to induce significant vasodilatation [15].

\section{Discussion}

In the current study, using a cutoff value of 0.84 for the subendocardial relative perfusion index provided an ROC AUC of 0.88 for predicting an FFR less than or equal to 0.80 , which is in the range of the previously reported values for adenosine perfusion MRI [16-18]. We nevertheless observed that the relative myocardial perfusion index during maximal hyperemia is the best perfusion MRI predictor for FFR-defined inducible coronary ischemia on a per-lesion basis. Indeed, when compared with the risk-uncorrected hyperemic perfusion and the perfusion reserve index, the relative perfusion index showed a significantly improved correlation with the FFR value. Our observations are in line with those of previous studies evaluating the functional significance of coronary artery stenosis by myocardial blood flow estimates obtained with PET $[19,20]$. In those studies, correction of the hyperemic blood flow in risk areas according to that of remote normal ar- 
TABLE I: Patient Demographics and Cardiovascular Risk Factors According to Myocardial Ischemia as Defined by Catheter Fractional Flow Reserve (FFR)

\begin{tabular}{l|c|c|c}
\hline \multicolumn{1}{c|}{ Patient Characteristics } & $\begin{array}{c}\text { No Ischemia } \\
\text { (FFR }>0.80)(n=31)\end{array}$ & $\begin{array}{c}\text { Ischemia } \\
(\text { FFR } \leq 0.80)(n=15)\end{array}$ & $p$ \\
\hline Age (y), mean \pm SD (range) & $61 \pm 9(44-80)$ & $62 \pm 9(48-80)$ & 0.763 \\
Sex, no. of patients & & & 0.867 \\
Male & 22 & 11 & \\
Female & 9 & 4 & 0.229 \\
Body mass index, mean \pm SD (range) & $29 \pm 5(21-39)$ & $27 \pm 3(24-35)$ & 0.672 \\
Resting heart rate (beats/min), mean \pm SD & $68 \pm 13(51-100)$ & $67 \pm 8(54-81)$ & \\
(range) $_{\text {Family history of coronary disease }}$ & $9(29)$ & $3(20)$ & 0.513 \\
Personal history of coronary disease & $3(10)$ & $5(33)$ & 0.047 \\
Diabetes mellitus & $10(32)$ & $2(13)$ & 0.170 \\
Current tobacco smoker & $10(32)$ & $7(47)$ & 0.305 \\
Elevated blood lipid profile & $23(74)$ & $13(87)$ & 0.336 \\
Systemic hypertension & $25(80)$ & $8(53)$ & 0.053 \\
Agatston coronary calcium score, median & $225(139-480)$ & $465(109-578)$ & 0.561 \\
(interquartile range) $^{\mathrm{b}}$ & & & \\
\hline
\end{tabular}

Note-Except where noted otherwise, data are number (percentage) of patients.

aWeight in kilograms divided by the square of height in meters.

${ }^{\mathrm{b}}$ Four men were not included because of prior coronary stenting.

TABLE 2: Correlations Between Fractional Flow Reserve (FFR) Values and Subendocardial Perfusion Indexes on MRI

\begin{tabular}{l|c|c|c|c|c}
\hline $\begin{array}{c}\text { Subendocardial Perfusion } \\
\text { Index }\end{array}$ & Mean \pm SD & $r$ & $p$ & $\begin{array}{c}\text { Best Cutoff Value for } \\
\text { FFR } \leq 0.80\end{array}$ & AUC \\
\hline Stress maximal upslope & $0.17 \pm 0.04$ & 0.273 & 0.06 & 0.16 & 0.69 \\
Perfusion reserve & $1.23 \pm 0.53$ & 0.288 & 0.04 & 1.25 & 0.67 \\
Relative perfusion & $0.17 \pm 0.04$ & 0.587 & $<0.001$ & 0.84 & 0.88 \\
\hline
\end{tabular}

eas also resulted in better correlation with the FFR value, compared with other uncorrected estimates, such as stress-only myocardial blood flow or stress-to-rest ratios.

In contrast to previous reports [7-10], we found a poor correlation between the FFR value and both the uncorrected perfusion and the perfusion reserve index in risk myocardium. Regarding pathophysiology, discordances of $30-40 \%$ are observed between FFR and the methods interrogating both epicardial vessel and microvasculature, such as coronary flow reserve and uncorrected perfusion MRI. These discordances are due to several factors, such as the prevalence of microvascular disease or diffuse coronary disease [21]. The proportion of subjects with longstanding symptoms in different study samples theoretically influences the correlations between FFR and stress-perfusion MRI. Coronary microcollateral vessels may develop after long-duration flow reduction, especially when regular physical exercise is performed [22], although this was shown recently to affect the FFR value marginally [23]. A possibly higher proportion of microvascular disease in our study sample as compared with the previous studies should be contemplated as well. In addition, our findings might be due to the narrower FFR range (0.60-0.98) of the lesions in our study compared with previous studies that included lesions with much lower FFR values [7-10, 24]. Independently from the circulatory physiology, the signals as measured by perfusion MRI and the myocardial blood flow are heterogeneous by essence, with a variability of up to $35 \%$ of normal myocardial perfusion in highly controlled settings [6]. Finally, a certain number of patients may have no sufficient response from the vasodilator. These technical failures probably explain why, even after correction by using the relative perfusion index, the correlation with the FFR value remains moderate in our study $(r=0.59)$. Indeed, the three false-negative cases in predicting FFR less than or equal to 0.8 all involved patients who did not respond to adenosine. Together, both provide a clue to the low diagnostic value of uncorrected perfusion MRI for FFR-defined myocardial ischemia, as reported in a recent trial [25], and indicate that relative perfusion index is the parameter with the greatest ability to control the confounders and the discrepancies between perfusion MRI and FFR-defined ischemia [17].

Our work also questions the respective prognostic importance of relative and uncorrected perfusion defects, because the use of an FFR less than or equal to 0.80 to determine the need for revascularization and decrease the rate of unnecessary procedures is increasingly debated [26]. It has been shown that uncorrected perfusion MRI defects may provide useful prognostic information regarding event-free survival in patients with ischemic heart disease [27-29], but the contribution of other causes of perfusion defect (e.g., microvascular or spastic diseases) to patient outcomes has not been established yet $[21,28,30]$. Therefore, the FFR less than or equal to 0.80 cutoff remains the most acknowledged determinant of cardiovascular events and death. Accordingly, our findings indicate that the relative perfusion index may be the most relevant prognostic parameter derived from stress perfusion MRI in the current clinical practice [31-33].

\section{Limitations}

There are limitations to our study, including the relatively small number of patients examined and its retrospective and observational nature. The use of intracoronary papaverine for invasive FFR measurements was different from the approach of the Fractional Flow Reserve Versus Angiography for Multivessel Evaluation studies [1, 14], although similar hyperemia can be obtained compared with IV adenosine administration [34]. Also, because a normal reference myocardial area is required as remote myocardium, our approach may be limited by both the fact that a less than $40 \%$ diameter stenosis on the feeding artery does not fully guarantee the control of the FFR confounders on maximal hyperemia, and that the approach is ineffective in patients with multivessel disease in a similar way to other diagnostic techniques $[11,19,20]$. Evaluation of the relative perfusion index also requires knowledge of the coronary artery anatomy. However, patients 


\section{Ghekiere et al.}

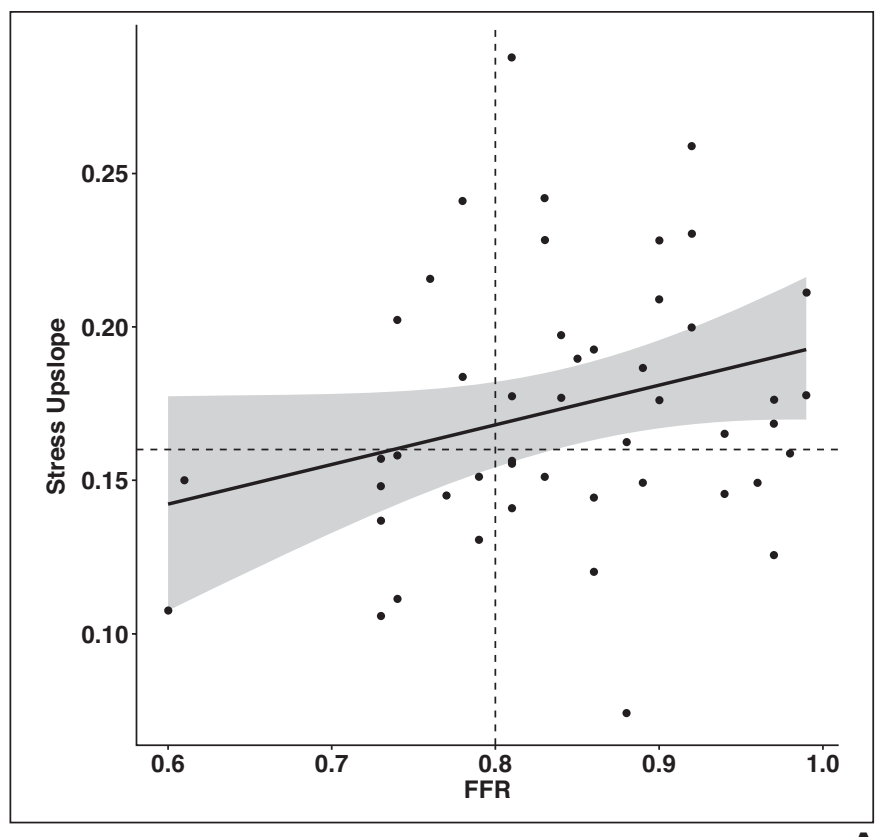

A

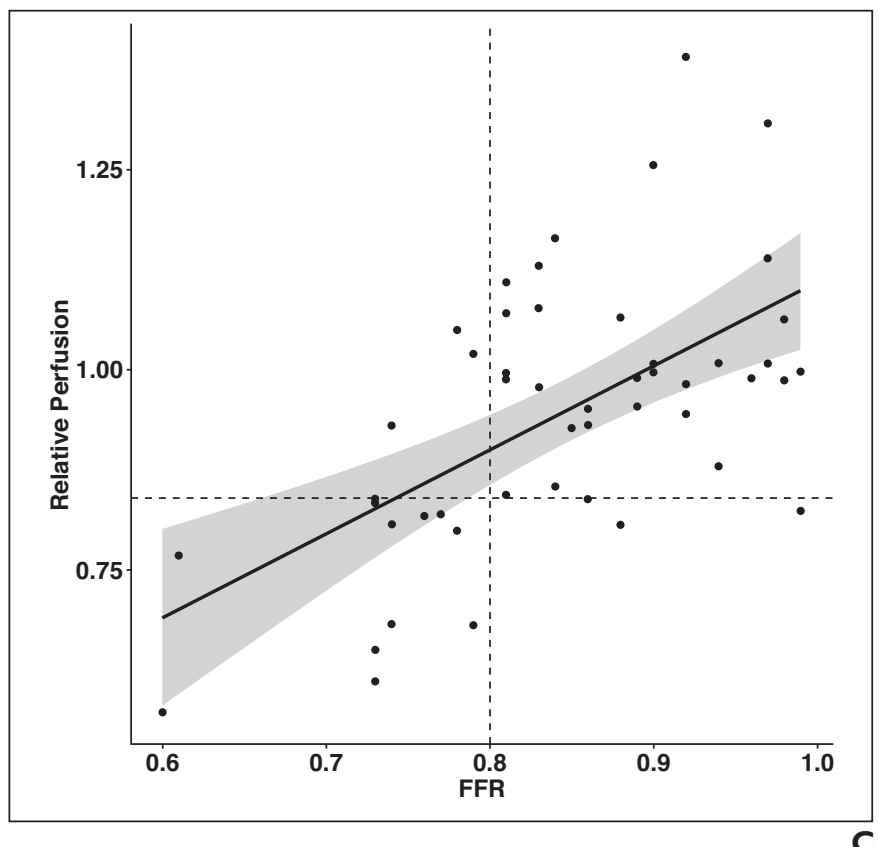

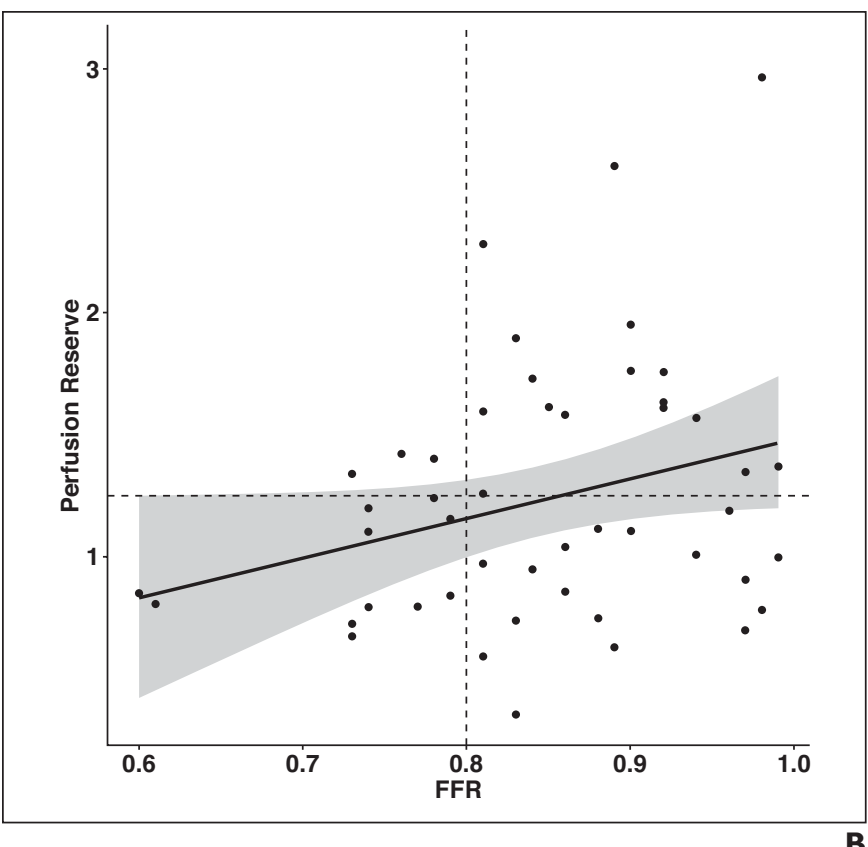

Fig. 3-Correlations between fractional flow reserve (FFR) and subendocardial perfusion MRI.

A-C, Graphs show that maximal upslope in risk area on stress (A) and perfusion reserve index (stress-to-rest upslopes) (B) were very weakly correlated with FFR value, whereas relative perfusion index (risk-to-remote upslopes on stress) (C) had improved correlation. All regression lines (solid lines) are given with their $95 \%$ Cls (shaded areas), and dashed lines represent respective cut-offs for myocardial ischemia. Dots denote individual data points.

TABLE 3: Diagnostic Values of Subendocardial Perfusion Indexes on MRI for Fractional Flow Reserve $\leq \mathbf{0 . 8 0}$ Coronary Artery Stenosis

\begin{tabular}{|c|c|c|c|c|c|c|c|c|c|c|c|}
\hline $\begin{array}{l}\text { Coronary Artery } \\
\text { Stenosis }(n=49)\end{array}$ & \multicolumn{4}{|c|}{ No. of Findings } & \multicolumn{5}{|c|}{ No. of Findings/Total (\%) [95\% Cl] } & \multicolumn{2}{|c|}{ Likelihood Ratio (95\% CI) } \\
\hline Stress maximal upslope & 22 & 11 & 4 & 12 & $\begin{array}{c}22 / 34(65) \\
{[63-67]}\end{array}$ & $\begin{array}{c}11 / 15(73) \\
{[69-77]}\end{array}$ & $\begin{array}{c}22 / 26(85) \\
{[82-86]}\end{array}$ & $\begin{array}{c}11 / 23(48) \\
{[45-51]}\end{array}$ & $\begin{array}{c}33 / 49(67) \\
{[66-69]}\end{array}$ & $\begin{array}{c}2.43 \\
(2.36-2.50)\end{array}$ & $\begin{array}{c}0.48 \\
(0.47-0.49)\end{array}$ \\
\hline Perfusion reserve & 17 & 12 & 3 & 17 & $\begin{array}{c}17 / 34(50) \\
{[48-52]}\end{array}$ & $\begin{array}{c}12 / 15(80) \\
{[75-83]}\end{array}$ & $\begin{array}{c}17 / 20(85) \\
{[81-87]}\end{array}$ & $\begin{array}{c}12 / 29(41) \\
{[39-44]}\end{array}$ & $\begin{array}{c}29 / 49(59) \\
{[58-61]}\end{array}$ & $\begin{array}{c}2.50 \\
(2.42-2.59)\end{array}$ & $\begin{array}{c}0.62 \\
(0.62-0.63)\end{array}$ \\
\hline Relative perfusion & 12 & 12 & 3 & 3 & $\begin{array}{c}31 / 34(91) \\
{[89-92]}\end{array}$ & $\begin{array}{c}12 / 15(80) \\
{[75-83]}\end{array}$ & $\begin{array}{c}31 / 34(91) \\
{[89-92]}\end{array}$ & $\begin{array}{c}12 / 15(80) \\
{[73-83]}\end{array}$ & $\begin{array}{c}43 / 49(88) \\
{[86-89]}\end{array}$ & $\begin{array}{c}4.56 \\
(4.41-4.71)\end{array}$ & $\begin{array}{c}0.11 \\
(0.11-0.11)\end{array}$ \\
\hline
\end{tabular}

Note-A high likelihood ratio $(>10)$ is a good indicator for ruling in the ischemia, whereas a low likelihood ratio $(<0.1)$ is a good indicator for ruling out ischemia. TN $=$ true negative, $\mathrm{TP}=$ true positive, $\mathrm{FN}$ = false negative, $\mathrm{FP}=$ false positive, $\mathrm{NPV}=$ negative predictive value, $\mathrm{PPV}=$ positive predictive value. 
Perfusion MRI of FFR-Defined Ischemia

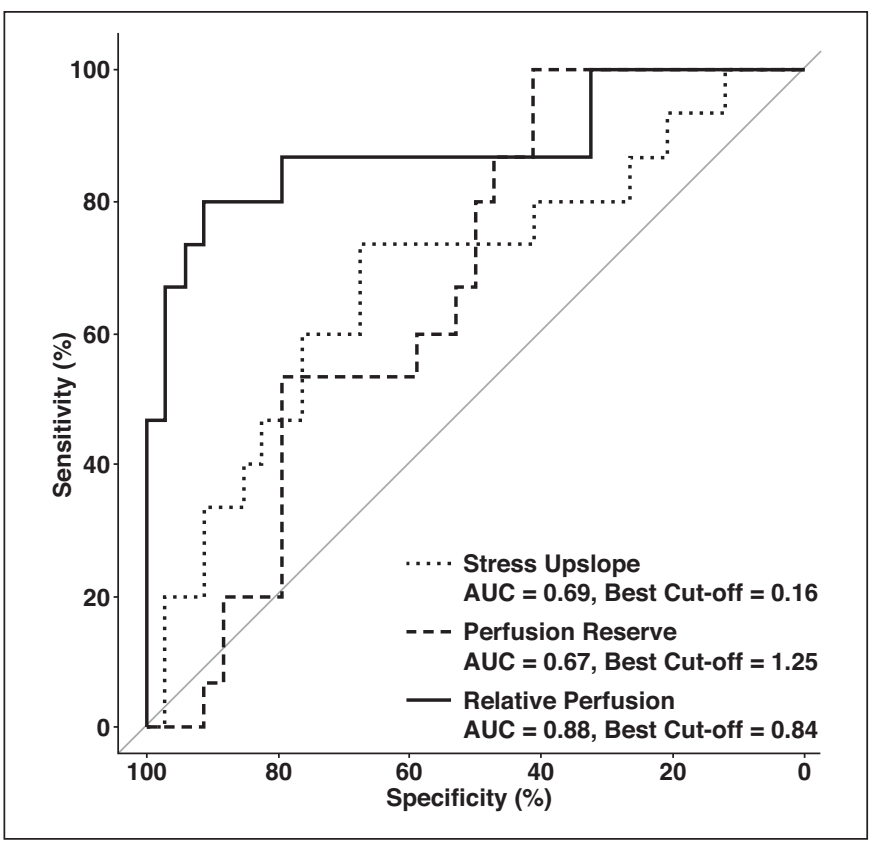

with obstructive coronary artery disease as diagnosed with coronary CT or invasive coronary angiography are increasingly observed in the daily practice. These patients often require additional functional imaging to assess myocardial ischemia, especially for intermediate-grade coronary stenosis [35].

\section{Conclusion}

Using perfusion MRI, assessment of the relative subendocardial perfusion index provides the best prediction for lesion-specific ischemia as defined by FFR. Further studies with larger patient samples and hypothesis verification are needed to confirm these important preliminary findings, emphasizing that this index could be used to noninvasively determine the need for revascularization of coronary stenoses detected on other investigations such as CT or catheter angiographies.

\section{References}

1. De Bruyne B, Pijls NH, Kalesan B, et al.; FAME 2

Trial Investigators. Fractional flow reserve-guided PCI versus medical therapy in stable coronary disease. N Engl J Med 2012; 367:991-1001

2. De Bruyne B, Baudhuin T, Melin JA, et al. Coronary flow reserve calculated from pressure measurements in humans: validation with positron emission tomography. Circulation 1994; 89:1013-1022

3. Tesche C, De Cecco CN, Albrecht MH, et al. Coronary CT angiography-derived fractional flow reserve. Radiology 2017; 285:17-33

4. Johnson NP, Gould KL, Di Carli MF, Taqueti VR. Invasive FFR and noninvasive CFR in the evalua-
Fig. 4-Graph of $\mathrm{ROC}$ analysis of subendocardial perfusion MRI indexes to determine fractional flow reserve less than or equal to 0.80 coronary stenoses.

tion of ischemia: what is the future? $J$ Am Coll Cardiol 2016; 67:2772-2788

5. Schuster A, Zarinabad N, Ishida M, et al. Quantitative assessment of magnetic resonance derived myocardial perfusion measurements using advanced techniques: microsphere validation in an explanted pig heart system. J Cardiovasc Magn Reson 2014; 16:82

6. Klocke FJ, Simonetti OP, Judd RM, et al. Limits of detection of regional differences in vasodilated flow in viable myocardium by first-pass magnetic resonance perfusion imaging. Circulation 2001; 104:2412-2416

7. Rieber J, Huber A, Erhard I, et al. Cardiac magnetic resonance perfusion imaging for the functional assessment of coronary artery disease: a comparison with coronary angiography and fractional flow reserve. Eur Heart J 2006; 27:1465-1471

8. Kühl HP, Katoh M, Buhr C, et al. Comparison of magnetic resonance perfusion imaging versus invasive fractional flow reserve for assessment of the hemodynamic significance of epicardial coronary artery stenosis. Am J Cardiol 2007; 99:1090-1095

9. Kirschbaum SW, Springeling T, Rossi A, et al. Comparison of adenosine magnetic resonance perfusion imaging with invasive coronary flow reserve and fractional flow reserve in patients with suspected coronary artery disease. Int $J$ Cardiol 2011; 147:184-186

10. Lockie T, Ishida M, Perera D, et al. High-resolution magnetic resonance myocardial perfusion imaging at 3.0-Tesla to detect hemodynamically significant coronary stenoses as determined by fractional flow reserve. J Am Coll Cardiol 2011; 57:70-75

11. Hussain ST, Chiribiri A, Morton G, et al. Perfu- sion cardiovascular magnetic resonance and fractional flow reserve in patients with angiographic multi-vessel coronary artery disease. J Cardiovasc Magn Reson 2016; 18:44

12. Cerqueira MD, Weissman NJ, Dilsizian V, et al. Standardized myocardial segmentation and nomenclature for tomographic imaging of the heart: a statement for healthcare professionals from the Cardiac Imaging Committee of the Council on Clinical Cardiology of the American Heart Association. Circulation 2002; 105:539-542

13. Tarroni G, Corsi C, Antkowiak PF, et al. Myocardial perfusion: near-automated evaluation from contrast-enhanced MR images obtained at rest and during vasodilator stress. Radiology 2012; 265:576-583

14. Tonino PA, De Bruyne B, Pijls NH, et al. Fractional flow reserve versus angiography for guiding percutaneous coronary intervention. $N$ Engl $J$ Med 2009; 360:213-224

15. Manisty C, Ripley DP, Herrey AS, et al. Splenic switch-off: a tool to assess stress adequacy in adenosine perfusion cardiac MR imaging. Radiology 2015; 276:732-740

16. Li M, Zhou T, Yang LF, Peng ZH, Ding J, Sun G. Diagnostic accuracy of myocardial magnetic resonance perfusion to diagnose ischemic stenosis with fractional flow reserve as reference: systematic review and meta-analysis. JACC Cardiovasc Imaging 2014; 7:1098-1105

17. Takx RA, Blomberg BA, El Aidi H, et al. Diagnostic accuracy of stress myocardial perfusion imaging compared to invasive coronary angiography with fractional flow reserve meta-analysis. Circ Cardiovasc Imaging 2015; 8:e002666

18. Danad I, Szymonifka J, Schulman-Marcus J, Min JK. Static and dynamic assessment of myocardial perfusion by computed tomography. Eur Heart J Cardiovasc Imaging 2016; 17:836-844

19. Lee JM, Kim CH, Koo BK, et al. Integrated myocardial perfusion imaging diagnostics improve detection of functionally significant coronary artery stenosis by ${ }^{13} \mathrm{~N}$-ammonia positron emission tomography. Circ Cardiovasc Imaging 2016; 9:e04768

20. Stuijfzand WJ, Uusitalo V, Kero T, et al. Relative flow reserve derived from quantitative perfusion imaging may not outperform stress myocardial blood flow for identification of hemodynamically significant coronary artery disease. Circ Cardiovasc Imaging 2015; 8:e002400

21. van de Hoef TP, van Lavieren MA, Damman P, et al. Physiological basis and long-term clinical outcome of discordance between fractional flow reserve and coronary flow velocity reserve in coronary stenoses of intermediate severity. Circ Cardiovasc Interv 2014; 7:301-311

22. Mobius-Winkler S, Uhlemann M, Adams V, et al. Coronary collateral growth induced by physical 


\section{Ghekiere et al.}

exercise: results of the Impact of Intensive Exercise Training on Coronary Collateral Circulation in Patients With Stable Coronary Artery Disease (EXCITE) Trial. Circulation 2016; 133:14381448; discussion, 1448

23. Gould KL. Intense exercise and native collateral function in stable moderate coronary artery disease: incidental, causal, or clinically important? Circulation 2016; 133:1431-1434

24. Chiribiri A, Hautvast GL, Lockie T, et al. Assessment of coronary artery stenosis severity and location: quantitative analysis of transmural perfusion gradients by high-resolution MRI versus FFR. JACC Cardiovasc Imaging 2013; 6:600-609

25. Nissen L, Winther S, Westra J, et al. Diagnosing coronary artery disease after a positive coronary computed tomography angiography: the DanNICAD open label, parallel, head to head, randomized controlled diagnostic accuracy trial of cardiovascular magnetic resonance and myocardial perfusion scintigraphy. Eur Heart J Cardiovasc Imaging 2018; 19:369-377
26. Gaemperli O, Marsan NA, Delgado V, Bax JJ. The year in cardiology 2014: imaging. Eur Heart $J$ 2015; 36:206-213

27. Vincenti G, Masci PG, Monney P, et al. Stress perfusion CMR in patients with known and suspected CAD: prognostic value and optimal ischemic threshold for revascularization. JACC Cardiovasc Imaging 2017; 10:526-537

28. Hussain ST, Paul M, Plein S, et al. Design and rationale of the MR-INFORM study: stress perfusion cardiovascular magnetic resonance imaging to guide the management of patients with stable coronary artery disease. J Cardiovasc Magn Reson 2012; 14:65

29. Coelho-Filho OR, Seabra LF, Mongeon FP, et al. Stress myocardial perfusion imaging by CMR provides strong prognostic value to cardiac events regardless of patient's sex. JACC Cardiovasc Imaging 2011; 4:850-861

30. Zimmermann FM, Ferrara A, Johnson NP, et al. Deferral vs. performance of percutaneous coronary intervention of functionally non-significant coronary stenosis: 15-year follow-up of the
DEFER trial. Eur Heart J 2015; 36:3182-3188

31. Plein S, Motwani M. Fractional flow reserve as the reference standard for myocardial perfusion studies: fool's gold? Eur Heart J Cardiovasc Imaging 2013; 14:1211-1213

32. Depta JP, Patel JS, Novak E, et al. Outcomes of coronary stenoses deferred revascularization for borderline versus nonborderline fractional flow reserve values. Am J Cardiol 2014; 113:1788-1793

33. Gould KL, Johnson NP. Physiologic stenosis severity, binary thinking, revascularization, and "hidden reality”. Circ Cardiovasc Imaging 2015; 8:e002970

34. Park JY, Lerman A, Herrmann J. Use of fractional flow reserve in patients with coronary artery disease: the right choice for the right outcome. Trends Cardiovasc Med 2017; 27:106-120

35. Groothuis JG, Beek AM, Brinckman SL, et al. Combined non-invasive functional and anatomical diagnostic work-up in clinical practice: the magnetic resonance and computed tomography in suspected coronary artery disease (MARCC) study. Eur Heart J 2013; 34:1990-1998 